\title{
Paraísos, guerras y objetos voladores no identificados: análisis crítico del discurso dominante sobre amor, sexo y género en la versión española de cuatro populares revistas de ocio ${ }^{1}$
}

\author{
Berta GARCÍA FAET \\ Universitat Pompeu Fabra \\ bgarciafaet@gmail.com
}

Recibido: 15.06 .2013

Aceptado: 09.11.2013

\begin{abstract}
RESUMEN
El propósito de este artículo es llevar a cabo un análisis crítico del discurso dominante, a través del estudio de la elección léxica (metáforas y campos semánticos), sobre amor, sexo y estereotipos de género, en cuatro populares revistas de ocio recientemente publicadas en España. En primer lugar, se estudiará la división de género de las subtareas del amor y del sexo (la seducción como el rol de los hombres y la fidelización del cliente, el de las mujeres); en segundo lugar, se examinará de qué modo estos dos fenómenos son objeto de una ideología normativa connotada según la idea de la ciencia esotérica; en tercer lugar, se investigaran las tres metáforas predominantes (el amor y el sexo como paraíso, guerra y objeto) y su relación con los modelos de mujer y de hombre que se preconizan. Finalmente, se dotará de coherencia a todo el análisis según lo que llamaremos "romanticismo instrumental".
\end{abstract}

Palabras clave: Metáforas, Campos semánticos, Amor, Sexo, Estereotipos de género.

\section{Paradises, wars and ufos: critical analysis of dominant discourses} about love, sex and gender in the spanish version of four popular magazines

\begin{abstract}
The purpose of this paper is to carry out a critical analysis of the dominant discourses about love, sex and gender stereotypes, by studying the lexical choices (metaphors and semantic fields) in four popular magazines recently published in Spain. Firstly, I will analyze the gender division of love \& sex "works" (seduction, for men, and customer loyalty, for women). Secondly, I will examine the way in which these two phenomena are the object of some normative ideology, in relation to the idea of esoteric science; thirdly, I will take into consideration the three predominant metaphors (love and sex live paradise, war and object) and their relation with the leading models of women and men. Lastly, I will offer my conclusion on what I will call "instrumental romanticism".
\end{abstract}

Key words: Metaphors, Semantic Fields, Love, Sex, Gender Stereotypes.

$1^{\urcorner}$Este artículo ha sido realizado con el apoyo de Obra Social Fundación "la Caixa" (Beca de Excelencia otorgada para el curso académico 2011/2012) 


\section{INTRODUCCIÓN}

¿Se excitan las mujeres modernas con el prototipo de macho alfa protector? ¿Son las mujeres modernas unas geishas del amor, serviciales y complacientes? ¿Siguen buscando a un varón sustentador (bread-winner)? ¿Cuáles son las estrategias más eficaces para conservar al hombre bien atado? ¿Adoran los hombres contemporáneos a las mujeresmamá o, más bien, a las mujeres-niña? ¿Siguen buscando casarse con una muñeca, como antaño? ¿Cuáles son las palabras mágicas para cautivar a una mujer?

Estas, por supuesto, no son las preguntas que pretende responder este estudio: la pregunta fundamental que aspiramos a responder, precisamente, qué significan este tipo de preguntas. ¿Qué presuponen, qué dan por sentado, qué prescriben, cuál es el modelo de conducta que venden como apropiado, normal o natural? El propósito de este trabajo es, pues, doble: por un lado, queremos llevar a cabo un análisis crítico de los discursos dominantes sobre amor y sexo que se dan en una muestra de cuatro revistas de ocio publicadas en España; por otro, complementamos este análisis crítico poniéndolo en relación con los estereotipos de género que aparecen en estas mismas publicaciones.

¿Cómo se supone que son -0 , más bien, cómo se supone que deberían ser- las mujeres y los hombres de hoy en día cuando se trata de amor y sexo? ¿Qué se entiende, en concreto, por amor y sexo? ¿Cómo se experimentan estos fenómenos o, mejor dicho, cómo se indica que deben experimentarse? Y, en definitiva, ¿cómo encajan las concepciones hegemónicas de amor y sexo con los estereotipos hegemónicos de mujer y hombre? Estas sí son las cuestiones que guían nuestra investigación, cuyo objetivo último es el de poder contribuir a la reflexión crítica sobre el discurso normativo que difunde el poder sobre sentimentalidad e identidades (Van Dijk, 1999: 26-27). Pero, ¿por qué es tan relevante -académica y políticamente- este análisis?

En el ámbito de los estudios feministas, los análisis críticos del discurso sobre las construcciones de la dicotomía género versus sexo, y los estereotipos asociados a ella, son muy habituales. Todos ellos muestran invariablemente la preeminencia del modelo social patriarcal: por un lado, las personas son divididas binariamente en "mujeres" y "hombres"; por otro, se rellenan sustantivamente esas etiquetas de mujer y hombre con los atributivos normativos que los miembros del conjunto tienen que cumplir y que, por lo demás, van en especial detrimento de la autonomía y el bienestar de aquellas personas esencializadas en la etiqueta de "mujer". En concreto, el patriarcado dota de derechos, privilegios y una posición dominante a lo que él mismo califica y constriñe en la categoría de hombres; mientras que dota a las mujeres de atributos secundarios y menores; así como de, en general, una posición de sumisión, objetivación o, cuando menos, minusvaloración (Beauvoir, 2011). 
Pues bien: detectamos un cierto vacío en el estudio de cómo estos estereotipos de género propios del patriarcado interactúan o se encajan con el discurso dominante sobre lo que deben ser el amor y el sexo ${ }^{2}$. Es esta laguna la que abordamos en este artículo. ¿Qué metáforas y qué campos semánticos dan cuenta de cómo se normativizan las relaciones sentimentales de nuestro tiempo? ¿Qué nos dicen esas metáforas y campos semánticos sobre la relación amoroso-sexual entre la "mujer" normativa y el "hombre" normativo, entre la "auténtica mujer" y el "auténtico hombre", entre una "mujer normal" y un "hombre normal"? ¿Cómo hay que amar, cómo hay que practicar sexo, cómo hay que cautivar, cómo hay que conservar a la pareja a nuestro lado? ¿Qué está permitido, qué está prohibido, qué es voluntario y qué es obligatorio?

\section{POSTULADOS TEÓRICOS.}

Nuestra mirada parte de cuatro esferas diferentes: análisis crítico del discurso, estudios culturales de género, sociología de las emociones y lingüística cognitiva.

Desde el análisis crítico del discurso, consideramos que hay que trascender el análisis ordinario del discurso para tratar de destapar las estructuras de poder subyacentes. Así, el análisis crítico del discurso está interesado en estudiar "el modo en que el abuso del poder social, el dominio y la desigualdad son practicados, reproducidos y ocasionalmente combatidos por los textos y el habla en el contexto social y político." Explicitando y sacando a la luz que "las relaciones de poder son [en parte, aunque no únicamente] discursivas" y que "el discurso hace un trabajo ideológico", nuestro objetivo al adscribirnos a este enfoque es el de esclarecer estas relaciones de poder y este trabajo ideológico intensivo que realizan (Van Dijk, 1999: 23-24).

En cuanto a los estudios culturales de género, nos basamos sobre todo en el trabajo de Judith Butler $(1990,2001)$ y su consideración del género (y del propio sexo) como una construcción social e ideológica que se puede $-\mathrm{y}$ se debe, crítica, paródica y/o subversivamente- moldear; el género es, de hecho, un "artefacto"3.

Butler denuncia cómo el poder se ancla en los discursos dominantes del género (que son binarios, dicotómicos, hetero-normativos y patriarcales) y cómo los sujetos pueden, si bien no sin esfuerzo, negarse a aceptar esa naturalización. No en vano el género (como

$2\urcorner$ Sí existe una amplia literatura sobre los estereotipos de género en las revistas para mujeres. Ver, por ejemplo, López Rodríguez, Irene (2007): "The representation of women in teenage and women's magazines: recurring metaphors in English", Estudios Ingleses de la Universidad Complutense vol. 15, pág. 16.

3 Preciado, Beatriz (Entrevista a): "Es urgente e imprescindible en el s. XXI una rebelión de los cuerpos", en Diagonal 130/131 (2010). 
cualquier otro componente de la identidad) es ante todo un "efecto performativo" (Ribas y Todolí, 2008: 156), es decir, pura repetición, puro acto, y no "esencia", "naturaleza" o "biología".

La sociología de las emociones remite a una nueva escuela sociológica (que apenas cuenta con veinticinco años de existencia) orientada, como su propio nombre indica, al estudio de una dimensión humana que, en la sociología clásica, había sido negligente o intencionadamente olvidada: los afectos, las pasiones y los sentimientos (Bericat, 2000). Estos sí se habían tratado en psicología y en filosofía, pero no en sociología, esto es, no puestos en relación con las estructuras sociales. Los tres autores pioneros de esta disciplina son Thomas J. Scheff, Theodore K. Kemper y Arlie Hochshild, pero aquí nos apoyaremos solamente en esta última y en Eva Illouz.

Para Hochshild (2008: 159), el objeto de la sociología de las emociones es "teorizar sobre todo aquello que se hace evidente cuando hacemos la simple asunción de que lo que sentimos es tan importante como lo que pensamos o lo que hacemos para el resultado de la interacción social." Las emociones tienen un importante valor sociológico porque las emociones están cargadas y recargadas de significados. Esta autora (2008: 160) distingue entre tres dimensiones: la normativa, la expresiva y la política:

"La primera se fundamenta en el hecho de que las normas sociales no sólo se aplican a la conducta y al pensamiento, sino también a las emociones. Podemos hablar, entonces, de la existencia de «normas emocionales» (feeling rules) (...) Las normas emocionales constituyen un modo de control social que definen lo que debemos sentir en diversas circunstancias, indicando cuál es el sentimiento apropiado y deseable en cada caso."

De Illouz, tomamos la tesis principal de su monumental trabajo "Consuming the Romantic Utopia: Love and the Cultural Contradictions of Capitalism" (1997), a saber: el amor y el sexo en el capitalismo se basa, por un lado, en la idea romántica de "gratuidad" y unión perfecta de los amantes (que recoge, entre otras, la metáfora del amor-sexo como paraíso) y, por otro lado, esta idea de "gift" queda totalmente corrompida con la "instrumentalización" que domina la mayoría de las relaciones sentimentales, basadas en el propio interés y en el propio beneficio (idea que recoge, entre otras, la metáfora del amor-sexo como guerra y como objeto):

"Although the cultural motif of love as pure 'waste and gift' is withering away, the various metaphors of love as an intense force are anchored in social and economic practices of waste, a pure expenditure of self and wealth for the sake of an organic bond. On the other hand, the love-as-work model is rooted in the mutually profitable exchange of contractual relationships." (1997: 196) 
Por último, desde la lingüística cognitiva, en este trabajo consideramos que las metáforas están en la misma estructura lingüística de nuestro pensamiento. George Lakoff (Lakoff y Johnson, 2012) se inserta en la tradición filosófico-lingüística que sostiene que no podemos acceder directa u ontológicamente a "la verdad de las cosas", a causa del "filtro distorsionador" del lenguaje; es más, todo lenguaje humano opera vía tropos antropomórficos ${ }^{4}$ :

"Nuestro sistema conceptual ordinario, en términos del cual pensamos y actuamos, es fundamentalmente de naturaleza metafórica." (2012:39)

Y define la lógica de la metáfora como: "el extender y experimentar un tipo de cosa en términos de otra." (2012: 41)

Lakoff sostiene que la mayoría de las metáforas que usamos (entre otras, las estructurales y las ontológicas), reflejan el sistema de valores de una cultura determinada. Por ejemplo: en la cultura occidental contemporánea, las metáforas de "el tiempo es oro" y "la discusión es una batalla" recogen determinados valores que construyen nuestra manera de vivir. $\mathrm{O}$, dicho con otras palabras:

"Some metaphors not only have a cognitive basis, but also are culturally motivated, that is, they reflect the attitudes and beliefs held by a particular community. (...) Most metaphors are not neutral in their evaluative stance, but are charged with an ideological or attitudinal component (...)" (2012: 18)

Es por esto que buena parte de nuestro estudio se centra en el análisis de la elección semántica: las metáforas -aparentemente irrelevantes o, como mucho, meramente poéticas-, y los campos semánticos, en realidad dicen mucho sobre la ideología que promueve el discurso del poder.

\section{PROPUESTA METODOLÓGICA}

Nuestro estudio consta de tres partes:

(a) En primer lugar, analizamos la importancia relativa de los dos subtemas básicos detectados en el macro-tema de "amor y sexo" (a partir de ahora, A\&S), a saber,

$4\urcorner$ Esta tesis es profundamente nietzscheana. Ver Nietzsche, Friedrich (2012): 'Sobre la verdad y mentira en sentido extramoral' y otros fragmentos de filosofia del conocimiento. Edición preparada por Manuel Garrido. Madrid: Tecnos, y Lizcano, Emmanuel (1999): "La metáfora como analizador social”, Empiria 2, pág. 35. 
seducción y fidelización del cliente. Este análisis se realiza en función del géneromodelo (mujer $u$ hombre) al que se dirigen los textos seleccionados.

(b) En segundo lugar, examinamos la normatividad omnipresente en el macro-tema A\&S. Analizamos cómo la presencia de los campos semánticos de la ciencia y la técnica implican concebir el amor y el sexo como "saberes objetivos". La utilización de estos campos semánticos nos indica que se está sosteniendo la tesis implícita de que, cuando se trata de amor y sexo, hay una forma correcta y una forma incorrecta de hacer las cosas.

(c) En tercer lugar, analizamos la relación del contenido normativo concreto en torno a A\&S con los estereotipos de género. Por un lado, explicitamos los estereotipos de género que se dan en los textos estudiados. Por otro lado, determinamos cuál es, según estos mismos textos, la forma correcta y la forma incorrecta de hacer las cosas cuando se trata de amor y sexo, y ello en función del género-modelo (mujer u hombre) del público destinatario de cada revista. Veremos que lo que se vende es una concepción del amor y del sexo que varía según el género al que se esté dirigiendo el texto: la presencia de los campos semánticos del paraíso, en el caso de las mujeres, y de la guerra, en el caso de los hombres, así lo sugiere.

(d) Las revistas seleccionadas para este estudio son Ragazza, Cosmopolitan, Marie Claire y Men's Health, en sus números de marzo de 2012 y en sus versiones españolas. Las tres primeras están destinadas a un público femenino (adolescente, joven y joven/adulto respectivamente), mientras que la cuarta está dirigida a un público masculino (joven/adulto). Se trata de una muestra limitada, por cuestiones de espacio, pero suficientemente representativa, del tipo de literatura que nos interesa según nuestro objeto de estudio (a saber, el discurso predominante sobre A\&S y su relación con los estereotipos de género), en las revistas multidisciplinares más leídas en España a día de hoy. Según la Oficina de la Justificación de la Difusión, el promedio de tirada mensual fue, en 2010, de 60.417 para Ragazza, 233.150 para Cosmopolitan, 150.159 para Marie Claire y 84.209 para Men's Health.

(e) Por supuesto, las revistas seleccionadas tratan temas variados que no pueden subsumirse en el macro-tema A\&S (como moda, tendencias, belleza, salud, deporte, psicología y espectáculos; de ahí que las hallamos denominado genéricamente como "revistas de ocio"). Por ello, hemos seleccionado únicamente los artículos que tratan específicamente de amor y/o sexo, y el número total de artículos asciende a 15. De ellos, 3 son de Ragazza, 9 de Cosmopolitan, 1 de Marie Claire y 2 de Men's Health. 


\section{CÓMO SEDUCIR Y CÓMO FIDELIZAR.}

Dejando momentáneamente a un lado un tercer cajón de sastre en el que podemos incluir los casos más idiosincráticos, distinguimos dos importantes subtemas: seducción y fidelización del cliente. A continuación mostramos una tabla con las revistas, los títulos de los artículos que pertenecen a cada una de ellas, y el subtema en el que encajan.

\begin{tabular}{|c|c|c|}
\hline Ragazza & In y Out del ligue & Sec ıcción \\
\hline & Caso real: "Me escapé de casa or amor" & ( ( 'tro) \\
\hline & $\begin{array}{l}\text { 3. ¿Quieres llevarle al cielo? Des ubre sus } \\
\text { puntos } \mathrm{G}\end{array}$ & Fidelizaci $\mathrm{n}$ del cliente \\
\hline \multirow[t]{9}{*}{ I osmopolitan } & 1. Qué decir después del sexo & Fidelizaci $\mathrm{n}$ del cliente \\
\hline & Cómo consigo no discutir con 1 & Fidelizaci $\mathrm{n}$ del cliente \\
\hline & Infidelidad femenina & $(1$ tro $)$ \\
\hline & Momentos en los que es buenc estar celosa & Fidelizaci $\mathrm{n}$ del cliente \\
\hline & Prohibido no tocar & $\begin{array}{l}\text { Fidelizaci n del cliente/ } \\
\text { Sec ıcción }\end{array}$ \\
\hline & ¿Estarías dispuesta a llegar al 1 mite? & $\begin{array}{c}\text { Fidelizaci } \mathrm{n} \text { del cliente/ } \\
\text { Sec ıcción }\end{array}$ \\
\hline & ¿Por qué él, por qué ella? & $(1$ tro $)$ \\
\hline & ¿Te quedarías con tu plan B? & ( 1 tro $)$ \\
\hline & Qué odian que lleves en la can $\mathrm{l}$ & $\begin{array}{c}\text { Fidelizaci n del cliente/ } \\
\text { Sec ıcción }\end{array}$ \\
\hline Tarie Claire & Más sexo, menos mimos & $(1$ tro $)$ \\
\hline \multirow[t]{2}{*}{ Ien's Health } & Seducción virtual & Sec ıcción \\
\hline & 36 maneras de ligarte a esta ch $\mathrm{za}$ & Sec ıcción \\
\hline
\end{tabular}

Con seducción nos referimos a aquellas acciones (indicadas o aconsejadas en los artículos seleccionados) destinadas a atraer romántica y sexualmente a la persona deseada; la RAE define el verbo "ligar" como "entablar relaciones amorosas o sexuales pasajeras". Hacemos, pues, hincapié en el primer contacto y en el corto/medio plazo, con independencia de que, finalmente, sí pueda darse un largo plazo.

Sin embargo, la fidelización del cliente contiene dos matices relevantes: en primer lugar, no se trata solamente de conseguir una relación romántica y/o sexual en un momento dado sin más preocupación por lo que suceda después, sino de mantener una relación romántico-sexual a lo largo del tiempo. En otras palabras, implica una fidelidad sostenida. En segundo lugar, utilizamos el término "fidelización del cliente" para resaltar, mediante la inclusión del vocablo "cliente", perteneciente al mundo económico, que cuando hay fidelización hay, básicamente, un intercambio de lo que podríamos calificar como "servicios". 
La seducción es el primer paso y la fidelización del cliente es el segundo; primero hay que captar la atención y, después, mantenerla. Por eso hay una linealidad temporal entre ambos fenómenos, si bien es cierto que no siempre es fácil separarlos (de ahí que algunos artículos deban ser caracterizados como pertenecientes a ambos subtemas, si bien a nuestro entender siempre hay uno que predomina). En la práctica, la cuestión es dirimir si se trata de conquistar (lo que es una acción más bien agresiva, pues se pasa de la nada del antes de la seducción al algo del después de la seducción) o de conservar (lo que supone, al contrario, un trabajo más constante y regular). Metafóricamente, podríamos conceptuar la seducción como el acto de encender un fuego y el de la fidelización del cliente, como el de mantenerlo.

Veamos en unos gráficos cuál es la importancia relativa de ambos fenómenos en nuestra selección en relación al género de los lectores destinatarios

\section{Fuente: elaboración propia}

Lo primero que llama la atención de la muestra es que los dos extensos artículos de Men's Health, la revista para hombres, tratan de la seducción, mientras que en el conjunto de las revistas femeninas (especialmente en Cosmopolitan), el subtema estrella es el de la fidelización del cliente.

La seducción del hombre a la mujer que se preconiza en Men's health se plasma en una serie de consejos para ligar: así, por ejemplo, se explica qué es "lo mejor para conocer nuevas amantes", "lo mejor para encontrar pareja" y "lo mejor para calentar el ambiente" (MH, texto $\left.{ }^{\circ} 1\right)$.

En las revistas femeninas, si bien también se habla de la seducción necesaria del primer contacto ("Ficha las nuevas tendencias a la hora de seducir y... iponte al día!", en $\mathrm{R}$, texto $\mathrm{n}^{\circ} 1$ ), la fidelización del cliente, esto es, cómo mantener al hombre satisfecho para que no se vaya, es sin duda el objetivo clave al que se dedican la mayoría de los artículos (6 de 9 artículos de Cosmopolitan, o 7 del total de 13 artículos de las revistas femeninas). Veamos algunos ejemplos (de aquí en adelante, todas las cursivas y negritas son nuestras):

-“CCómo consigo no discutir tanto con él? [Espacio] ¿Te peleas mucho con tu chico? ¿Os pasáis el día como el perro y el gato? Pues he aquí las fórmulas para vivir en paz y armonía." (C, texto $\left.\mathrm{n}^{\mathrm{o}} 2\right)$

-“Quieres que tu chico se sienta aún más unido a ti??” (C, texto $\left.\mathrm{n}^{\circ} 5\right)$

-“[Tienes que] generar una verdadera cercanía.” (C, texto $\left.\mathrm{n}^{\circ} 5\right)$ 
Berta García Faet $\quad$ Paraísos, guerras y objetos voladores no identificados: análisis crítico del discurso...

5) - "Esta cercanía física fomentará una conexión única entre los dos.” $\left(C\right.$, texto $n^{o}$

-“Detalles que no te conviene descuidar (si llevas mucho tiempo con él): la rutina es la peor enemiga de la pareja. [He aquí] unos consejos extra [para] mantener viva la llama de la pasión: depílate (unas pantorrillas peludas o un bigote de sargento pueden ser visiones descorazonadoras y síntomas de dejadez, sobre todo en una pareja de largo recorrido); cuida tu olor (...); no exhibas tus complejos (...); quítate los calcetines (...); cambia de peinado [ya que] cambiar tu pelo es una buena forma de sorprender a tu chico y romper la rutina. ¡Será casi como si estuviera con otra!" $\left(\mathrm{C}\right.$, texto $\left.\mathrm{n}^{\circ} 9\right)$

El hecho de que en la revista masculina predomine el cómo seducir y en las revistas femeninas el cómo fidelizar al cliente, tiene mucha relación con un hallazgo que comentaremos más adelante: la elección semántica. La seducción es activa, y el campo semántico de la guerra, así, se aplica a ella con una naturalidad que nos hace entender cuán bélica es determinada manera de entender el "ligue" masculino. La fidelización del cliente es un trabajo para alumnas aplicadas, constantes, atentas y solícitas; no resulta extraño que el campo semántico de la ciencia y la técnica y, por supuesto, el del paraíso, estén tan presentes en este subtema. Pero esto lo veremos después. De momento, destaquemos un ilustrativo fragmento que deja claro que la seducción es la tarea propia de los hombres y la fidelización, la de las mujeres:

"No olvides que en el reino animal la hembra despierta el magnetismo pero el macho es quien debe cazar a la presa. Sus instintos reproductivos no han cambiado desde los tiempos de las cavernas." (C, texto $\left.\mathrm{n}^{\circ} 5\right)$

Llama también la atención el cajón de sastre de los casos que no encajan estrictamente ni en el subtema de la seducción ni en el de la fidelización del cliente:

En primer lugar, es de destacar que hay dos artículos sobre la infidelidad (en ambos casos, como práctica considerada legítima), uno en Cosmopolitan y otro en Marie Claire; además, en un artículo de Men's Health ( $\mathrm{MH}$, texto $\left.\mathrm{n}^{\circ} 1\right)$, también se trata brevemente este fenómeno como "posibilidad".

En segundo lugar, hay tres artículos que sólo tienen en común la tesis implícita del romanticismo utópico (no es una sorpresa que estos tres artículos se encuentren en revistas para mujeres, Ragazza y Cosmopolitan): uno sobre los conflictos entre el amor verdadero y las opiniones de la familia que, en un prototípico final feliz, en última instancia, siempre acaban disolviéndose ( $\mathrm{R}$, texto $\mathrm{n}^{\mathrm{o}} 2$, con el persuasivo título de "Caso real: me escapé de casa por amor"); otro sobre qué hace que determinadas personas se atraigan $\left(\mathrm{C}\right.$, texto $\mathrm{n}^{\circ} 7$, con el título de "Por qué él, por qué ella"), en el que se invoca el poder de la química; y otro sobre la inconveniencia, tanto práctica como ética, de 
renunciar al auténtico amor y conformarse con menos $\left(\mathrm{C}\right.$, texto $\mathrm{n}^{\circ} 8$, con el título de " ¿ $\mathrm{Te}$ quedarías con tu plan B?").

Por el momento, del análisis de los subtemas y su relación con la concepción contemporánea de A\&S y los estereotipos de género, cabe extraer las siguientes conclusiones:

(1) el macro-tema de A\&S tiene dos ejes temáticos fundamentales, que se corresponden, además, con los "momentos" o "fases" de una relación: la seducción, o la "caza", y la fidelización del cliente, o el "servicio".

(2) los hombres se dedican básicamente a seducir. Se resalta su papel "activo", su "iniciativa" (al menos en la primera fase de una relación).

(3) las mujeres, si bien también sí seducen (en el sentido de que han de provocar que se dé una atracción hacia su persona, mostrándose atractivas), su papel en la primera fase es más "pasivo", "expectante" o "de cebo", esto es, ella es el objeto de todas las miradas pero espera pacientemente a que el hombre dé el primer paso. La mujer sólo tiene que "incitarlo", es decir, "hacer que ocurra". Su verdadero protagonismo está en la fidelización del cliente, en el medio y largo plazo.

(4) En definitiva, ellos emprenden la relación y ellas la cuidan. Ellos prenden la hoguera y ellas garantizan que el calor no se escape por la ventana.

\section{NORMATIVIDAD: HAY UNA MANERA CORRECTA DE AMAR, HAY UNA MANERA CORRECTA DE PRACTICAR SEXO}

Destacan dos campos semánticos muy significativos -el de la ciencia y la técnica, por un lado, y el del secreto, por otro- que están, en mayor o menor medida, presente en todos los artículos estudiados. ¿Por qué?

El campo semántico de la ciencia y la técnica nos indica que el universo de las relaciones personales (tanto si se resalta la faceta del amor como la faceta del sexo) es considerado un universo susceptible de ser conocido objetivamente $\mathrm{y}$, de hecho, controlado instrumentalmente. Es más, incluso lo que claramente no son sino opiniones subjetivas e indemostrables, se consideran verdaderas tesis morales. Así, se presupone que hay una manera correcta (científica y éticamente) de amar y de practicar sexo, de seducir y de fidelizar.

El campo semántico del secreto matiza esta concepción, y nos indica que el discurso dominante en las revistas estudiadas es el de que, no sólo A\&S son materia de conocimiento seguro; además, dicho conocimiento seguro es un privilegio de 
determinados iluminados. El universo amoroso-sexual, por lo tanto, es ciencia y es técnica... pero ciencia y técnica esotéricas. Por supuesto, los redactores de las revistas se auto-conciben como "maestros" y "expertos", mientras que las/os lectoras/es son tratados como "alumnos" o, a veces, como "iniciados".

En consecuencia, la "norma" (o, en otras palabras, lo que hay que hacer para hacerlo bien) y el "misterio" (a saber, la idea de que "lo que hay que hacer" es un secreto trascendental y elitista que sólo conocen unos pocos: un tesoro escondido que los maestros comparten, desinteresadamente, con sus alumnos) se solapan.

Veamos todo esto con más detalle.

\section{La norma}

La normatividad con la que se trata la cuestión A\&S se plasma en la elección semántica de palabras que evocan "objetividad", tanto científica como moral. Así, se habla de "estrategias que hay que seguir" o "instrucciones" o "datos útiles" o "buenos niveles". Todo ello implica suponer que existen auténticos expertos en amor y sexo porque, de hecho, el amor y el sexo son disciplinas de conocimiento. Seducir y fidelizar son acciones que se pueden hacer bien o mal, eficiente o ineficientemente. Las fórmulas correctas se pueden y se deben aprender. ¡Hasta se pueden determinar los pasos que hay que seguir para echar una mirada de infarto! Todo ello es posible sólo porque las mujeres y los hombres son de una manera determinada, definitiva y comprobable; sobre esta base segura, debemos instruirnos en la cuestión de cómo convivir. De ahí que se utilicen tanto los enunciados expositivos, los mandatos imperativos y las "mediciones". A continuación mostramos algunos ejemplos:

-Instrucciones paso por paso para "seducir con la mirada" (o "tres pasos básicos para cautivarle": "1. Clava la mirada (...); 2. Mantén la mirada (...); 3. Retira la mirada $(\ldots) "\left(C\right.$, texto $\left.{ }^{\circ} 5\right)$

-“Toma nota de [estos] tips.” (R, texto $\mathrm{n}^{\circ} 3$ )

-"No olvides acariciarle y besarle otras zonas sensibles (...). Subirás la temperatura sí o sí.” (R, texto n ${ }^{\circ}$ )

-“Déjate guiar por nuestro mapa masculino.” (R, texto $\mathrm{n}^{\mathrm{o}} 3$ )

-"Manual de hombres" $\left(\mathrm{C}\right.$, texto $\left.\mathrm{n}^{\mathrm{0}} 1\right)$

-“101 cosas sobre los hombres" $\left(\mathrm{C}\right.$, texto $\left.\mathrm{n}^{\mathrm{0}} 1\right)$

-“¿Qué [hay que] decir después del sexo? Tras un buen orgasmo, cualquier chico quiere (1) saber que te ha dejado satisfecha y (2) dormir 
como un tronco. Escoge una de estas frases [Uf, eso que me has hecho ha sido increíble; Cariño, ha sido maravilloso, me lo he pasado genial; ¡Guau, nunca en mi vida había sentido algo así!] y... ¡lo harás feliz!” $\left(\mathrm{C}\right.$, texto $\left.\mathrm{n}^{\mathrm{o}} 1\right)$

-“He aquí las fórmulas para...” (C, texto n $\left.{ }^{\mathrm{o}} 2\right)$

-"Diga lo que diga tu chico, no pierdas los papeles y permanece en tu sitio." (C, texto $\mathrm{n}^{\circ}$ )

-"Es recomendable que..." $\left(\mathrm{C}\right.$, texto $\left.\mathrm{n}^{\mathrm{o}} 2\right)$

-“Otro recurso muy eficaz es..." $\left(\mathrm{C}\right.$, texto $\left.\mathrm{n}^{\mathrm{0}} 2\right)$

-"Te invitamos a que te descargues la aplicación Test de compatibilidad de Tapcoder.” $\left(\mathrm{C}\right.$, texto $\left.\mathrm{n}^{\mathrm{o}} 2\right)$

-[Utiliza nuestro] "termómetro de nivel de celos justificado." (C, texto $\left.\mathrm{n}^{\circ} 4\right)$

“¿Quieres que tu chico se sienta aún más unido a ti? Prueba a...” (C, texto nº 5)

-“¿Quieres aprender a sacar el máximo partido de este acercamiento? Aquí tienes algunas sugerencias. ¡Funcionan!” $\left(\mathrm{C}\right.$, texto $\left.^{\circ}{ }^{\circ}\right)$

-"Acaricia el lóbulo de su oreja con los dedos pulgar e índice", "Acaricia su cabello con los dedos", o "Masajea su músculo trapecio suavemente." (C, texto $\mathrm{n}^{\circ}$ 5)

-"Prepárate para hacer cosas que normalmente sólo ves en las pelis, explora territorios desconocidos..." $\left(\mathrm{C}\right.$, texto $\left.\mathrm{n}^{\mathrm{o}} 6\right)$

-“Conviértete en una bad bad girl." (C, texto n ${ }^{\circ}$ )

-Lista de "propuestas eróticas", en las que se indica el "nivel de perversidad" $\left(\mathrm{C}\right.$, texto $\left.^{\circ} 6\right)$

-Hay "factores" que "determinan" el enamoramiento. (C, texto $\left.{ }^{\circ} 7\right)$

-Lista de "prendas espanta-hombres". (C, texto n ${ }^{\circ}$ 9)

-Lista de "cosas que sí proceden en la alcoba". (C, texto n 9)

-“Detalles que no te conviene descuidar..." (C, texto no 9)

-"Otro error es disfrazarte de stripper con ropa de sex shop que metería miedo al macho más curtido." $\left(\mathrm{C}\right.$, texto $\left.\mathrm{n}^{\circ} \mathrm{9}\right)$ 
Berta García Faet $\quad$ Paraísos, guerras y objetos voladores no identificados: análisis crítico del discurso...

-[He aquí una] “regla infalible”. (C, texto n 9)

-“Dato útil: el 75\% de los chicos...” (C, texto $\left.n^{\circ} 9\right)$

-Se puede determinar cuál es el "buen nivel de deseo sexual”. (MC, texto $\left.\mathrm{n}^{\circ} 1\right)$

-“Recuerda: muéstrate un poco pícaro...” $\left(\mathrm{MH}\right.$, texto $\left.\mathrm{n}^{\circ} 1\right)$

-"El siguiente paso es..." (MH, texto $\left.\mathrm{n}^{\mathrm{o}} 1\right)$

-“[He aquí] 36 maneras de ligarte a esta chica.” (MH, texto $\left.n^{\circ} 2\right)$

-“Usa la estrategia de los dos besos.” (MH, texto $\left.\mathrm{n}^{\mathrm{o}} 2\right)$

-“[He aquí las] seis palabras mágicas” ( $\mathrm{MH}$, texto n²)

La función persuasiva de este campo semántico científico-técnico (estrategias, instrucciones, datos, eficiencia, manual, fórmulas, errores, reglas infalibles, etc.) es evidente: envolver en un disfraz de objetividad toda esta serie de mensajes normativos (y, por tanto, ni científicos ni técnicos) que son, por el contrario, pura ideología.

\section{El misterioso y esotérico secreto.}

Pero este conocimiento objetivo, al que se llega mediante la ciencia y al que se puede controlar mediante la técnica, es en realidad un conocimiento misterioso y esotérico... Sólo unos pocos iluminados, los "maestros" o "expertos", conocen toda la verdad. Por suerte, se trata de maestros solidarios, siempre dispuestos a compartir su sabiduría. Veamos algunos ejemplos:

-“ A explorar!", [te mostramos] un tesoro escondido y un secreto al descubierto.” (R, texto $\left.\mathrm{n}^{\circ} 3\right)$

-"Este punto G está escondido y puede pasar desapercibido, pero nosotras te lo chivamos." (R, texto $\left.\mathrm{n}^{\circ} 3\right)$

-“En el próximo número te contaremos todos los secretos del cuerpo femenino." $\left(\mathrm{R}\right.$, texto $\mathrm{n}^{\mathrm{o}} 3$ )

-“¿Espíale! Descubre en estas páginas todos los secretos del universo masculino" $\left(\mathrm{C}\right.$, texto $\left.\mathrm{n}^{\mathrm{o}} 1\right)$

-"Puede que tu chico se esfuerce por limpiar su historial todos los días, pero Google nunca olvida. Estas son algunas de las (delatoras) búsquedas más realizadas por los hombres." (C, texto $\left.\mathrm{n}^{\mathrm{o}} 1\right)$ 
Berta García Faet Paraísos, guerras y objetos voladores no identificados: análisis crítico del discurso...

-“Sigue leyendo y lo sabrás.” (C, texto $\left.\mathrm{n}^{\mathrm{o}} 2\right)$

-"Desclasificamos archivos secretos con información de alto voltaje." (C, texto $\left.n^{0} 3\right)$

-“Reportaje sin censuras." (C, texto $\left.\mathrm{n}^{\mathrm{o}} 3\right)$ $\left.n^{0} 7\right)$

-“Te desvelamos los factores que se esconden tras la fecha de Cupido.” (C, texto

-“[Desciframos] esa ley 'sobrenatural' que hace que sea esa persona y no otra la que queremos a nuestro lado." (C, texto $\left.\mathrm{n}^{\mathrm{o}} 7\right)$

-“Se llegó a la conclusión de que la ciencia podía ser el foco que necesitábamos para terminar de iluminar el misterio de la atracción." $\left(\mathrm{C}\right.$, texto $\left.\mathrm{n}^{\mathrm{o}} 7\right)$

La función del campo semántico del secreto también es obvia: por un lado, aumentar el morbo y el sensacionalismo; por otro, generar gratitud por la solidaridad y la ayuda demostrada por los expertos, que desinteresadamente comparten su valioso saber. Así se crea la útil sensación de que "comprar esta revista sirve para algo".

\section{Las fuentes}

La supuesta objetividad del conocimiento científico-técnico que ofrecen los textos estudiados contrasta con su utilización difícilmente rigurosa de las fuentes. Los argumentos de autoridad que se invocan (que suelen remitirse a lo que se intenta hacer pasar por "ciencia" y "técnica") se basan en datos inexistentes o incorrectos, como cuando se califica de "muestra representativa" a (¡literalmente!) "un puñado de chicos" (ver $\mathrm{C}$, texto $\mathrm{n}^{\circ} 1$ ). Veamos algunos ejemplos de la nula seriedad de las fuentes empleadas:

-“Sabías que...? Normalmente, las relaciones sexuales duran alrededor de 15 minutos." (Sin fuente) $\left(\mathrm{R}\right.$, texto $\left.\mathrm{n}^{\circ} 1\right)$

-“El 69\% de los hombres piensan que..." (Sin fuente) $\left(C\right.$, texto $\left.n^{\circ} 1\right)$

-Porcentaje de "ciudades infieles" y de "nacionalidades infieles" (Sin fuente) $(C$, texto $\mathrm{n}^{\mathrm{o}} 3$ )

-“El 75\% de los chicos de los chicos aborrecen el camisón holgado estilo 'El exorcista' o 'La casa de la pradera"” (Sin fuente) $\left(\mathrm{C}\right.$, texto $\mathrm{n}^{\circ}$ 9)

-Testimonio de "Sheila (Alicante)" $\left(\mathrm{R}\right.$, texto $\left.\mathrm{n}^{\circ} 2\right)$ 
Berta García Faet $\quad$ Paraísos, guerras y objetos voladores no identificados: análisis crítico del discurso...

-Testimonio de "María (24 años)” (MC, texto n 1 )

-Testimonio de "Laura (abogada, 31 años)" (C, texto $\left.n^{\circ} 8\right)$

-“Según ashleymadson.com..." (C, texto n³)

-“Estudio de meetic.com” (MH, texto n $\left.{ }^{\mathrm{o}} 1\right)$

-Según "un reciente estudio realizado desde la web de Cosmopolitan..." (C, texto $\mathrm{n}^{\mathrm{o}} 1$ )

-Según “una rápida encuesta vía Facebook” $(\mathrm{MC}$, texto n 1)

-"Según un estudio de la Michigan University" (sin nombre ni enlace al estudio) $\left(\mathrm{C}\right.$, texto $\left.\mathrm{n}^{\circ} 2\right)$

-"Lo deja bien claro un estudio de la Universidad de California en Berkely" (sin nombre ni enlace al estudio) $\left(\mathrm{C}\right.$, texto $\left.\mathrm{n}^{\mathrm{o}} 2\right)$

-Datos del INE (sin nombre ni enlace al estudio) $\left(\mathrm{C}\right.$, texto $\left.\mathrm{n}^{\circ} 8\right)$

No deja de ser irónico entre contraste entre fondo científico y forma científica: las píldoras ideológicas se disfrazan de conocimiento, pero se disfrazan mal.

\section{AMOR Y SEXO PARA MÚJERES, AMOR Y SEXO PARA HOMBRES: EL PARAÍSO, LA GUERRA, EL OBJETO Y LOS ESTEREOTIPOS DE GÉNERO.}

El universo de las relaciones amorosas y sexuales se conceptualiza de manera diferente, en cuanto a expectativas y cualidades, según se trate de lecturas pensadas para mujeres o para hombres. Pero antes de analizar los estereotipos de género, comencemos con un examen de los tres campos semánticos más importantes: el de la guerra, el del paraíso y el del objeto. Cada uno de ellos produce su propia metáfora (y recordemos que las metáforas nos permiten pensar un fenómeno complejo en función de otro más simple o, al menos, más accesible): "EL AMOR ES UNA GUERRA", "EL AMOR ES UN PARAÍSO” y "EL AMOR ES UN OBJETO", respectivamente.

\section{El amor es una guerra}

La seducción es una batalla en la que hay ganadores y perdedores, aliados y enemigos, estrategias eficientes o ineficientes, resultados aceptables o inaceptables. Así, en los artículos de la revista que se dirige a un público masculino, Men's Health, se utilizan machaconamente los siguientes términos: "armas de seducción", "arsenal", "cómo disparar", "probabilidades de éxito", "tomar la delantera", "uso y abuso de la 
tecnología", "riesgos", "objetivos", "herramienta", "estrategia”, "evaluar", "tener vía libre" y "ganar".

\section{El amor es un paraíso (ardiente)}

Los artículos de las revistas femeninas (especialmente, de Cosmopolitan) están plagados de metáforas que vinculan A\&S con el paraíso, si bien se trata de un paraíso muy especial: un paraíso infernal. De la combinación entre la metáfora "EL AMOR ES UN PARAÍSO" y la de "EL AMOR Y EL SEXO SON FUEGO", se obtiene la metáfora de "EL AMOR ES UN PARAÍSO ARDIENTE". Basten los siguientes ejemplos como muestra:

-"[Esta caricia] les lleva directamente al éxtasis [y] les provoca un placer infinito." (R, texto $\left.\mathrm{n}^{\circ} 3\right)$

-"Innovar y sorprender son las claves para hacer que tu chico sienta escalofríos de placer." (C, texto $\left.\mathrm{n}^{\circ} 6\right)$

-"Perversiones que os harán tocar el cielo". (C, texto nº 6 )

-“Hazle vibrar como nunca lo has hecho." (C, texto $\left.\mathrm{n}^{\mathrm{o}} 6\right)$

-“iTu chico se derretirá vivo!” (C, texto no 9)

Esta elección semántica está claramente relacionada con el romanticismo utópico incluso místico- que impregna los artículos analizados: placer, felicidad, perfección, eternidad... Todo, de hecho, "cobra una nueva dimensión" (R, texto $\left.{ }^{\circ} 2\right)$. De ahí las menciones a "la media naranja" $\left(\mathrm{C}\right.$, texto $\left.\mathrm{n}^{\circ} 7\right)$, "el príncipe azul", "el hombre de tu vida" $\left(\mathrm{C}\right.$, texto $\left.\mathrm{n}^{\mathrm{o}} 8\right)$, los "besos que cambian la vida" $\left(\mathrm{MH}\right.$, texto $\left.\mathrm{n}^{\mathrm{o}} 2\right)$, y el inmortal poder del "corazón", que es, en definitiva, quien "lleva las riendas".

\section{El amor es un objeto.}

Tres atributos nos permiten captar esta metáfora: el objeto amado puede ser poseído; las formas del amor son susceptibles de estar o no a la moda; y la relación amorosa se basa en un intercambio.

Que la mujer es considerada tradicionalmente como un mero objeto ya lo sabíamos (como queda patente, por ejemplo, en la recomendación de "Escríbeme una canción y soy tuya", o en el comentario de que los cumplidos predisponen positivamente a las mujeres para el sexo; ver $\mathrm{MH}$, texto $\mathrm{n}^{\circ}$ 2); lo curioso es que también el hombre es, o debe ser, un objeto para la mujer... Esta metáfora queda especialmente plasmada en el artículo de Cosmopolitan que trata sobre la legitimidad moral de los celos (C, texto $\left.\mathrm{n}^{\circ} 4\right)$, donde 
se sostiene que los celos están justificados si hay "motivos". Es más, hay "momentos en los que es bueno estar celosa"... Por ejemplo, cuando la compañera de piso del novio de la chica en cuestión es un "pibón"; o cuando dicho novio se va "de despedida de soltero a un club de strip-tease"; o cuando el hombre se niega a confesar "quién le manda [al móvil] esos mensajes intempestivos".

El amor también es objeto porque se evalúa según esté o no a la moda, sobre todo en Ragazza, la revista para el público femenino adolescente:

-“Ficha las nuevas tendencias para ligar” (R, texto $\left.n^{\circ} 1\right)$

-“Pásate ya a la nueva generación de gadgets eróticos” $\left(\mathrm{R}\right.$, texto $\left.\mathrm{n}^{\mathrm{o}} 1\right)$

_"Eso de hacerse arrumacos en la última fila [del cine] ya no se lleva nada" (R, texto $\mathrm{n}^{\mathrm{o}} 1$ )

-“[A la hora de ligar,] olvídate de los bares, las discotecas y el curro. ¡Están pasados de moda!" (R, texto $\left.\mathrm{n}^{\mathrm{o}} 1\right)$

Finalmente, la relación de A\&S también se basa en un intercambio, y lo que prima es el propio interés, satisfacción y beneficio. Así, se justifica moralmente el recíproco acariciar de los respectivos egos (lo que se llama "la virtualización de los amores utópicos", en $\mathrm{MH}$, texto $\mathrm{n}^{\mathrm{o}}$ 1) y se deja claro que los hombres hacen regalos a sus amantes mujeres a cambio de sexo ("Los regalos más habituales que hacen los amantes son: escapadas, perfumes, lencería y juguetes sexuales", en $\mathrm{C}$, texto $\mathrm{n}^{\mathrm{o}} 3$ ). Y lo que es aún más significativo: los regalos que hacen los hombres a las mujeres no son útiles, sino fundamentalmente "bonitos"-superfluos, superficiales o, en definitiva, "femeninos"-.

Este canje, mutuamente beneficioso para ambas partes pero de manera diferente, aparece muy explícitamente, por ejemplo, en el siguiente "consejo" de una mujer a un hombre:

"Si me mandas un obsequio bonito al trabajo, te pondré a cien con un mensaje picante al móvil.” ( $\mathrm{MH}$, texto $\left.\mathrm{n}^{\circ} 2\right)$

A esta tripe concepción de A\&S como guerra, paraíso y objeto, tenemos que añadir el papel de los estereotipos de género. Todo ello en su conjunto nos dará una idea muy clara de la pregunta con la que comenzábamos este trabajo: ¿cuáles son, y como se relacionan entre sí, los discursos dominantes sobre amor, sexo y género que se difunden desde el mundo de las revistas de ocio femeninas y masculinas? La respuesta sólo sorprenderá a aquellos que creían que, en la larga lucha contra el sexismo, algo, aunque fuera poco, habíamos avanzado. 


\section{ESTEREOTIPOS DE GÉNERO: MUJERES VERSUS HOMBRES}

\subsection{La confrontación.}

Hay dos ideas que recorren, tanto implícita como explícitamente, la totalidad de los artículos examinados. Se trata de los presupuestos fundamentales que están en la raíz del enfoque hegemónico sobre $\mathrm{A} \& \mathrm{~S}$ entre mujeres y hombres que venimos estudiando:

(a) Las mujeres y los hombres son diferentes. Cada grupo es, para su contrario y hasta cierto punto, incognoscible: siempre habrá características de la naturaleza del sexo contrario que se nos escaparán, pues no formamos parte de lo mismo: somos, de hecho, como el "perro" y el "gato" (C, texto $\left.\mathrm{n}^{\circ} 2\right)$. Si ha cambiado algo en los últimos tiempos, es irrelevante: lo importante es que seguimos perteneciendo a dos planetas diferentes:

“¿Se han convertido ellos en ositos amorosos al tiempo que nosotras mutábamos en vampiresas ardientes? [A la pregunta de] '¿Qué haces si tu pareja te da mimos cuando tú le pides sexo?', 19 de cada 20 mujeres responden como María (24 años): 'El día que un tío me pida o dé cariñitos en vez de un buen revolcón, lo dejo.' Contra todo lo pregonado en las últimas décadas acerca de nuestra debilidad por las carantoñas, las palabras de amor y los largos preliminares, frente al directo 'al grano' [del] 'aquí te pillo, aquí te mato' masculino, ahora resulta que ni todos los hombres son de Marte ni todas las mujeres son de Venus. Y que la tendencia es más bien opuesta. Ha caído el primer mito: los hombres demandan más besos y caricias que las mujeres." (MC, texto $\left.\mathrm{n}^{\mathrm{o}} 1\right)$

Por lo demás, cultura y biología se solapan: en ningún momento hay un cuestionamiento teórico sobre la naturaleza -natural o construida- de esas supuestas diferencias fundamentales; y si hay que privilegiar alguna, se privilegia la mítica "diferencia sexual", pues los "instintos reproductivos no han cambiado desde los tiempos de las cavernas" (C, texto $\mathrm{n}^{\circ}$ 5). Las diferencias que se detectan se validan automáticamente. $\mathrm{O}$ dicho con otras palabras: se acepta el status quo sin cuestionarlo críticamente.

(b) Las auténticas mujeres y los auténticos hombres son, en ambos casos y sin ningún tipo de duda, heterosexuales. Todos los artículos de las revistas femeninas explican cómo seducir y, especialmente, cómo fidelizar a los hombres; los artículos de Men's Health, por su parte, explican cómo seducir a las mujeres. Es una vuelta a la estrategia medieval: las preferencias sexuales homo o bi no se mencionan porque -se sobreentiende- no existen.

El discurso dominante es, pues, hetero-normativo y está basado en una división binaria y dicotómica entre mujeres y hombres. Además de la heterosexualidad, ¿cuál es 
Berta García Faet $\quad$ Paraísos, guerras y objetos voladores no identificados: análisis crítico del discurso...

el contenido normativo de esa división? ¿Cómo deben ser las mujeres normales? ¿Cómo deben ser los hombres normales?

\subsection{Las mujeres: saquitos de hormonas con vocación pin-up}

Si tuviéramos que formarnos una idea de cómo son las mujeres de verdad en base a lo que se presupone o prescribe en los artículos estudiados, el resultado sería bastante contradictorio: como todo estereotipo, resulta, a la postre, incoherente. La mujer resulta ser, a la vez, niña, maestra, loca, sirvienta, vasallo, pura apariencia y objeto. Nótese que, en todo caso, todas estas metáforas se forman en contra de un otro que, por supuesto, es el hombre (que, se supone, representa la norma universal, racional y normal) (Beauvoir, 2011). A continuación, exponemos los ejemplos más claros de estas curiosas metáforas:

a) La mujer-niña (que busca a un padre o a un bread-winner): la mujer hace "travesuras", pues es como una muñequita excitante, una graciosa bad bad girl $(\mathrm{C}$, texto $\mathrm{n}^{\mathrm{o}} 6$ ); la mujer busca estabilidad y teme la soledad (C, texto $\left.\mathrm{n}^{\mathrm{o}} 8\right)$; la mujer desea casarse $\left(\mathrm{MH}\right.$, texto $\left.\mathrm{n}^{\mathrm{o}} 1\right)$; la mujer busca planes a medio y largo plazo, porque busca seguridad, mientras que el hombre busca planes a medio y corto plazo, porque busca placer ("Me da más seguridad un hombre que se prepara para el futuro pero vive el presente. No hable falta que yo entre en tus planes inmediatos, pero deberías dejar un poco de espacio para que yo me imagine colándome en ellos", $\mathrm{MH}$, texto $\mathrm{n}^{\circ} 2$ ).

b) La mujer-maestra (que disfruta mandando): la mujer es típicamente aleccionadora ("Sospecharé que estás haciendo lo que no debes. (...) No hagas que piense que me he equivocado", $\mathrm{MH}$, texto $\mathrm{n}^{\circ} 2$ ); la mujer es una profesora mandona, en el fondo, sexy y complaciente ("Tú eres la que mandas así que prohíbele que tenga una erección mientras bajas a su miembro. Como ha sido un chico muy malo, y la ha tenido, castígale. Tírale a la cama, sujeta sus manos y cabálgale sin penetración. (...) Eres la profe de mates, regálale un $69^{\prime \prime}, \mathrm{C}$, texto $\mathrm{n}^{\circ} 6$ ).

c) La mujer-loca (histérica, vanidosa e inestable): la mujer es egocéntrica, y su vanidad tiene tanta importancia para ella como el sexo, concretamente oral, para el hombre ("Para las mujeres, saber escuchar significa lo mismo que el sexo oral para los hombres: demuestra que [el otro] de verdad te importa", $\mathrm{MH}$, texto $\mathrm{n}^{\mathrm{o}} 2$ ); la mujer es envidiosa e inconsistente, mientras que el hombre es más simple y está más equilibrado ("Haz cosas por mí delante de mis amigas. Así será más difícil sacarte pegas cuando te hayas ido. (...) A veces me siento como si tuviera varias personalidades. Adapta la tuya en consecuencia", $\mathrm{MH}$, texto $\mathrm{n}^{\mathrm{0}} 2$ ).

d) La mujer-sirvienta (cuya vocación lógica es estar al cuidado de su hogar y de la felicidad de su hombre): la mujer desea darle placer al hombre y, de hecho, su autoestima está directamente relacionada con su capacidad para provocar satisfacción ( “QQuieres 
llevarle al cielo? (...) [Esto] les lleva directamente al éxtasis (...) [Esto] les provoca un placer infinito", $\mathrm{R}$, texto $\mathrm{n}^{\mathrm{o}} 3$ ); la mujer es más cuidadosa, y está al servicio del marido ("Arréglale el cuello de la camisa o estira alguna arruga de su ropa. (...) Sois un equipo. (...) Observa a los entrenadores de fútbol... ¿Te has fijado que a menudo masajean los trapecios de sus jugadores antes de que salgan al campo? Cuando tu chico se siente agotado, necesita que te conviertas en su 'coach' personal", $\mathrm{C}$, texto $\mathrm{n}^{\circ}$ 5); la mujer es la reina de la cocina y, por lo tanto, se impresiona mucho si el hombre le enseña algún plato nuevo ("Ofrécete a cocinar. Me dejarás impresionada si me enseñas alguna receta que no conozca", $\mathrm{MH}$, texto $\mathrm{n}^{\circ}$ 2); la mujer es la reina de la higiene, y valora mucho la capacidad de limpieza y orden en un hombre, hasta el punto de que esa cualidad la enamora y embelesa ("Antes de verlo, su amiga ya le había contado que estaba encantada con él: se desenvuelve de maravilla entre los fogones y es más limpio que un jaspe", C, texto $\mathrm{n}^{\mathrm{o}} 7$ ).

e) La mujer-vasallo (que está bajo la protección de su señor, un respetable aunque un poco bruto- macho alfa): a la mujer le gusta, o bien el macho alfa ("No sabemos lo que tiene el look de leñador, pero cuando cierto tipo de hombre se lo pone, parece que te está diciendo sin palabras algo como 'Nena, me gustaría hacer cosas muy sucias contigo', $\mathrm{C}$, texto $\mathrm{n}^{\mathrm{o}} 1$ ); o bien, el caballero superior y condescendiente con los débiles o con los jerárquicamente inferiores a él.

Vale la pena detenerse brevemente en este punto: el respeto de la mujer hacia su persona se liga a su capacidad de mandar, de resolver problemas, de ser asertivo y de tener autoridad. A cambio, la mujer le proporciona cariño, lo que sin duda está relacionado con la metáfora de "EL AMOR ES UN OBJETO". En otras palabras, el amor se basa en un intercambio:

"Si perdonas la torpeza de un camarero, sonríes al cansado cajero de un banco o le ofreces el asiento a una mujer embarazada en el tren, automáticamente pasaré a considerarte un macho alfa."

"Cuanto más rápido seas capaz de llamar la atención del camarero, más respeto te tendré".

."[Si tengo algún problema práctico, como que la puerta de mi armario chirría o que tengo que cambiar la pila del reloj], soluciónalo antes de que te lo pida. Ver que arreglas algo me resulta atractivo." (todos, $\mathrm{MH}$, texto $\mathrm{n}^{\mathrm{o}} 2$ )

f) La mujer-pura apariencia (que es en lo que consiste la esencia de la feminidad): las chicas van con las chicas y hacen cosas de chicas ("Cuando acabe la bronca, pon música relajante, tipo chill out, y desconecta, o sal a correr por el parque, o llama a una amiga para desahogarte o vete de compras", $\mathrm{C}$, texto $\mathrm{n}^{\circ} 2$ ); la mística de la feminidad es un hecho incontrovertible que se da hasta en las situaciones límite ("Hasta para engañar 
somos femeninas: (...) las mujeres necesitamos que se nos corteje y halague (...) Las mujeres infieles se ponen muy guapas al sentirse deseadas. El juego de la infidelidad hace que les brillen los ojos, que sus gestos sean más seductores y que se vistan y maquillen más cuidadosamente", $\mathrm{C}$, texto $\mathrm{n}^{\circ} 3$ ); la feminidad se basa en la omnipresencia del complemento ("pídele que te quite las medias de rejilla y la liga (...) Usa tu barra de labios (...) Ponte una peluca sexy, un maquillaje atrevido y un vestido insinuante", C, texto $\left.\mathrm{n}^{\circ} 6\right)$.

g) La mujer-objeto (que irradia magnetismo y que puede ser poseído): la mujer es un objeto de deseo que mezcla pureza y lujuria ("[El blanco] transmite una sensación que mezcla pureza y lujuria. Y si rematas el conjunto con unas medias de rejilla blancas, pues apaga ¡Y vámonos! Este explosivo estilismo transformará tu cuerpo en un irresistible objeto de deseo", C, texto $\mathrm{n}^{\circ}$ 9); la ropa es el envoltorio del regalo, de la ofrenda, que es el cuerpo de la mujer. El siguiente fragmento deja claro que decadencia de este envoltorio/ofrenda, que es la apariencia femenina, es la causa típica de las rupturas sentimentales:

"Un tópico muy extendido dice que al hombre no le importa lo que la mujer lleve puesto, sino lo que hay debajo de su ropa. Puede ser. Pero a veces el envoltorio es tan espantoso y tan anti-erótico que puede provocar un irreversible y patético gatillazo. Es más, es probable que cierto tipo de prendas 'de alcoba' sean responsables del alto porcentaje de separaciones que se producen últimamente: cuando una chica empieza a descuidar su aspecto en la cama... es síntoma inequívoco de que la relación está en franca decadencia. $O$ tal vez la relación vaya bien y es la chica la que, por despiste o torpeza, no sabe vestirse (o desvestirse) para amar." $\left(\mathrm{C}\right.$, texto $\left.{ }^{\circ} 9\right)$

Pasemos ahora a los estereotipos masculinos que, como veremos, son igual de inconsistentes que los que acabamos de examinar.

\subsection{Los hombres: sementales simplones obsesionados con su virilidad}

El hombre (siempre en contraste con la mujer) es, a la vez, un niño, un estratega y una bestia.

a) El hombre-niño (que hace caso a la maestra y, a la vez, se siente fascinado por su cuerpo): el hombre, si ama a la mujer, "se porta requetebién" (C, texto $\left.\mathrm{n}^{\circ} 4\right)$; al hombre le embruja que la mujer "sugiera" ("[Nos gustan las] prendas que no exhiben, sino que sugieren y resaltan tu deliciosa anatomía", C, texto ${ }^{\circ} 9$ ).

b) El hombre-estratega (que, para seducir, no se muestra tal y como es, sino tal y como debe mostrarse): el hombre no es tímido, pero se "disfraza" de tímido para conseguir ligar ("Cuéntame un secreto que me enseñe tu lado sensible, aunque se note que intentas disimular tu incomodidad al confesarlo", $\mathrm{MH}$, texto $\mathrm{n}^{\circ} 2$ ); el hombre se lanza al amor como a la guerra (aquí la relación con la metáfora "EL AMOR ES UNA GUERRA" es más que evidente). Veamos algunos ejemplos de esto último: 
"Pero esas armas las tienes tú y cualquier otro mortal. Así que la clave está en saber cómo disparar."

“Recuerda: pon 'me gusta' [en su Facebook] cuando cuelgue alguna cosa en su muro, para demostrar que te interesa y tenéis gustos similares."

"Recuerda: emplea los DM [mensajes directos y privados entre dos usuarios] para pedir una cita con esa chica que a priori te interesa. Pero, por si acaso se decepciona, no compres el anillo de boda."

"El siguiente paso es el intercambio de números de móvil para seguir ligando por Whatsapp." (todos, $\mathrm{MH}$, texto $\mathrm{n}^{\mathrm{o}} \mathrm{1}$ )

c) El hombre-bestia (que es vanidoso, promiscuo, asertivo y superficial):

"Tras un buen orgasmo, cualquier chico quiere (1) saber que te ha dejado satisfecha y (2) dormir como un tronco", "A los hombres les gusta escuchar que son únicos". $\left(\mathrm{C}\right.$, texto $\left.\mathrm{n}^{\mathrm{o}} 1\right)$

"A muchos chicos les cuesta que les toquen esa zona [los pezones] por prejuicios (pueden llegar a pensar que son menos varoniles).” (R, texto $n^{\circ} 3$ )

"Si quieres que tu chico se comporte como el rey de la selva, apoya tu mano en el pecho y acaríciaselo lentamente. Notarás que empieza a sentirse más seguro más macho- y que poco a poco se excita. (...) [Esto] despertará su instinto de protección." (C, texto $\left.\mathrm{n}^{\mathrm{o}} 5\right)$

"Los hombres necesitan el contacto con las mujeres para reafirmar su masculinidad. Este sencillo gesto [de apoyar tu mano es su pecho] es especialmente efectivo, ya que él no tiene senos y tú sí, por lo que estarás remarcando la diferencia sexual que existe entre ambos. (...) Al rozar su pecho le estarás diciendo: tú eres el hombre y yo la mujer. Esto despertará su instinto de protección y deseo de forma inmediata, porque conseguirá que su cuerpo libere dopamina." $\left(\mathrm{C}\right.$, texto $\left.\mathrm{n}^{\mathrm{o}} 5\right)$

-"Poco a poco irás ganando espacio en la discusión y aplacando la ira de tu chico.” $\left(\mathrm{C}\right.$, texto $\left.\mathrm{n}^{\mathrm{o}} 2\right)$. Tienen peor mal humor por causas biológicas: “¿Sabías que los hombres tienden a acumular más adrenalina que las mujeres? El trapecio es una de las zonas que más agarrotamiento afronta, por lo que un masaje suave en este músculo le ayudará a calmarse." $\left(\mathrm{C}\right.$, texto $\left.\mathrm{n}^{\circ} 5\right)$

"No exhibas tus complejos: a ningún hombre le gusta estar todo el día escuchando letanías como 'estoy gordísima', 'tengo un montón de granos', o 'me siento fea'. Todo esto mata la libido.” (C, texto $\left.\mathrm{n}^{\circ} 9\right)$ 
"Lo de [hacer] el amor con los calcetines puestos horroriza a los hombres desde la Prehistoria." (C, texto $n^{\circ}$ 9)

La mujer normativa y el hombre normativo, como vemos, encajan entre sí: sin duda tales monstruos están hechos el uno para el otro... Siempre que el amor y el sexo sean paraíso, guerra y objeto; siempre que el amor y el sexo sean algo tan romántico y, a la vez, $\tan$ instrumental.

\section{CONCLUSIONES}

9.

Comenzábamos este artículo con una serie de preguntas retóricas: ¿siguen buscando las mujeres al legendario macho alfa? ¿Siguen los hombres queriendo adecuarse dicho ideal? ¿Les gusta a las mujeres ser unas enfermeras solícitas? ¿Sigue siendo su sueño el encontrar un hombre respetable, buen amante y trabajador, como Dios manda, para casarse y ser felices? ¿Cómo se atrapa a un hombre en nuestra red de tela de araña? ¿Buscan los hombres una mamá a la que obedecer o una hijita a la que hacer arrumacos, sentada sobre sus fuertes rodillas? Mama o baby? ¿Qué hay que hacer para derretir a una mujer?

La respuesta, si nos atenemos al discurso que se desprende de los artículos analizados, es un rotundo y triste sí. No queda claro si se trata de un "ser" o de un "deber ser"; en cualquier caso, esta distinción -como la de "innato" y "construido", "biológico" y "cultural"- no parece ser del interés de los redactores: la aceptación del status quo (sea éste el que sea) es automática.

Nuestro objetivo era llevar a cabo un análisis crítico del discurso dominante sobre amor, sexo y género en algunas de las revistas de ocio más representativas de este tipo de literatura masiva que se consume actualmente en España. $\mathrm{O}$, en otras palabras, queríamos examinar las concepciones hegemónicas de amor y sexo, y su relación con los estereotipos más hegemónicos, que pudieran desprenderse de la lectura atenta de estos altavoces sociales que son las revistas Ragazza, Cosmopolitan, Marie Claire y Men's Health.

¿Por qué? Porque la opresión tiene un lenguaje, y todo cambio requiere de una toma de conciencia.

El resultado es descorazonador: los estereotipos de género tradicionales no se distinguen demasiado de los modelos patriarcales clásicos; las "normas emocionales" que proclaman están profundamente connotadas por la ideología patriarcal, y las metáforas dominantes reflejan a la perfección la perfección tóxica de esos valores. 
Por lo demás, encajan como anillo al dedo en el ideal contemporáneo de amor y sexo que resulta ser, a la vez, romántico-utópico y mercantil-instrumental. Por un lado, se supone que el amor es felicidad, paz, proyecto, solidaridad. Por otro, el amor se basa en una relación de interés y de división del trabajo.

Ofrecemos, para finalizar, un decálogo de conclusiones:

1. El modelo patriarcal sigue, en lo fundamental, incólume: distingue binaria y dicotómicamente entre mujeres y hombres, y dota de un contenido sexista tradicional y hetero-normativo a ambos conjuntos.

2. La concepción hegemónica del tándem amor y sexo entiende a éstos, en primer lugar, como un campo de conocimiento objetivo, a pesar de la escasa rigurosidad de las fuentes a las que apela. Los campos semánticos de la ciencia y la técnica indican que el amor y el sexo son disciplinas científico-técnicas con el matiz misterioso-esotérico de que este conocimiento objetivo sólo lo poseen unos pocos: los expertos o maestros. Todo ello, combinado, permite pensar el amor y el sexo como norma: hay una forma correcta y una forma incorrecta de hacer las cosas.

3. La concepción hegemónica del tándem amor y sexo divide este universo en dos subtareas: seducción y fidelización del cliente.

4. Además, los denota según tres metáforas: "paraíso", "guerra" y “objeto".

5. La tarea de las mujeres es la de fidelizar al cliente: su papel de "cebo" es pasivo-magnético, y su papel de "cuidadora" encaja con su tarea de "hacer que la relación funcione". De ahí la importancia del campo semántico del paraíso: es trabajo de la mujer hacer que la relación sea celestial. Esta compleja relación se presupuestos se recoge en la metáfora "EL AMOR ES UN PARAÍSO (ARDIENTE)".

6. La tarea de los hombres es la de seducir: su papel de "cazador" es activobélico. Su trabajo es el de comenzar la relación llevando la iniciativa y asumiendo cualquier tarea que requiera autoridad, asertividad y control. De ahí la importancia del campo semántico de la guerra, pues, como el amor y el sexo son disciplinas de conocimiento objetivo, es posible idear estrategias para vincular medios y fines. La metáfora "EL AMOR ES UNA GUERRA" recoge esta idea.

7. El carácter de romanticismo utópico del modelo de capitalismo patriarcal se manifiesta en el campo semántico del paraíso y en algunos elementos del estereotipo de mujer.

8. El carácter del mercantilismo instrumental de dicho modelo queda recogido en el campo semántico del objeto, fundamentalmente en la metáfora de "EL AMOR ES UN 
OBJETO (QUE SE PUEDE INTERCAMBIAR)". El amor no es, por tanto, "gratuidad" y "gift", sino, antes bien, una cómoda y utilitarista intersección de intereses individuales.

9. El estereotipo de mujer la entiende a ésta como una mezcla de niña, maestra, loca, sirvienta, vasallo, pura apariencia y objeto. Las reminiscencias de la misoginia tradicional son muy claras.

10. El estereotipo de hombre lo entiende a éste como una mezcla de niño, estratega y bestia. Estos calificativos no dejan a los hombres necesariamente en un buen lugar, pero desde luego -y tal y como la lógica patriarcal dicta- al menos no son objetos sino, claramente, sujetos.

En definitiva, se proclama que mujeres y hombres vienen de planetas distintos y muy remotos entre sí; sin embargo, están destinados a unirse, a dividirse las tareas del amor y del sexo, a convivir en el paraíso y en la guerra.

En este artículo hemos analizado críticamente esta suposición, que consideramos perteneciente a una ideología sexista deplorable. Ni las mujeres, ni los hombres, ni el amor, ni el sexo, son o deben ser objetos, o tratados como objetos. Ni siquiera como preciosos y sugerentes objetos voladores no identificados.

\section{BIBLIOGRAFÍA}

BEAUVOIR, S. (2005, 2011): El segundo sexo. Madrid, Cátedra.

BERICAT, E. (2000): "La sociología de la emoción y la emoción en sociología”, Papers 62: $145-176$.

BUTLER, J. (1990): Gender Trouble. Feminism and Subversion of Identity. New York, Routledge.

BUTLER, Judith (2001): Cuerpos que importan. Barcelona, Paidós.

HOCHSCHILD, A. R. (2008): La mercantilización de la vida intima. Apuntes de la casa $y$ del trabajo. Madrid, Katz Editores.

ILLOUZ, Eva (1997): Consuming the Romantic Utopia: Love and the Cultural Contradictions of Capitalism. University of California Press.

LAKOFF, G. y JOHNSON, M. (2012): Metáforas de la vida cotidiana. Madrid, Cátedra.

LIZCANO, E.1 (1999): "La metáfora como analizador social”, Empiria 2: 29-60.

LÓPEZ RODRÍGUEZ, I. (2007): "The representation of women in teenage and women's magazines: recurring metaphors in English", Estudios Ingleses de la Universidad Complutense vol. 15: 15-42.

MILLETT, K. (2010): Política sexual. Madrid, Cátedra.

NIETZSCHE, F.h (2012): 'Sobre la verdad y mentira en sentido extramoral' y otros fragmentos de filosofía del conocimiento. Edición preparada por Manuel Garrido. Madrid: Tecnos. 
PRECIADO, Beatriz (Entrevista a): "Es urgente e imprescindible en el s. XXI una rebelión de los cuerpos", en Diagonal 130/131 (2010): http://www.diagonalperiodico.net/Es-urgente-e-imprescindible-en-el.html (último acceso 26/10/2012)

RIBAS, M. y TODOLÍ, J. (2008): "La metáfora de la mujer objeto y su reiteración en la publicidad", Discurso \& Sociedad, Vol. 2(1): 155-169.

VAN DIJK, T. (1999): “El análisis crítico del discurso”, Anthropos 186: 23-36. 